\title{
Révision de la taxe sur la valeur ajoutée: simplifier, oui ... mais pas à n'importe quel prix!
}

Il y a plus de deux ans que le corps médical se préoccupe de la révision de la taxe sur la valeur ajoutée (TVA): en avril 2006, la FMH a élaboré une prise de position à ce sujet à l'intention de la commission d'experts «Spori». Suite de quoi, elle a répondu en juillet 2007 à la procédure de consultation organisée par le Département fédéral des finances. Et voici quelques jours, la FMH a participé à une audition de la Commission de l'économie et des redevances du Conseil national, qui avait pour objet le message du Conseil fédéral sur la simplification de la TVA. La partie A de ce message propose plus de 50 mesures visant à remanier entièrement la loi sur la TVA. La partie B prévoit l'introduction d'un taux de TVA unique de 6,1\% et la suppression de la plupart des exceptions fiscales existant aujourd'hui.

L'opinion de la FMH sur ce message du gouvernement est claire: le corps médical estime qu'il est nécessaire de simplifier la procédure en matière de TVA. Cependant, $l^{\prime}$ «exception Santé» - il s'agit en fait de toute une série d'exceptions dans le domaine de la santé et du social ancrées dans l'article 18 de l'actuelle loi sur la TVA - doit être maintenue. De nombreux arguments sociopolitiques et économiques s'opposent en effet à la suppression de cette exception:

- Un assujettissement des traitements médicaux à la TVA entraînerait une augmentation des coûts de la santé de plus de cent millions de francs. Les primes d'assurance maladie connaîtraient une hausse supplémentaire supérieure à $4 \%$, ce qui nécessiterait un correctif sociopolitique pour les ménages aux revenus les plus modestes.

- La suppression proposée de la plupart des exceptions fiscales générerait quelque 30000 nouveaux assujettis à la TVA. Cela leur occasionnerait une charge administrative additionnelle considérable, de même qu'à leurs associations et à l'administration.

- En comparaison avec l'UE, où les traitements de médecine humaine sont exclus du champ de la TVA, la suppression de l'«exception Santé» créerait un important désavantage pour la Suisse dans ce secteur de l'économie.
- Le dégrèvement ultérieur d'impôt prévu pour les nouveaux assujettis, destiné à compenser le désavantage qu'ils subiraient pour n'avoir pas pu déduire l'impôt préalable sur leurs investissements antérieurs, pèserait sur les finances fédérales.

- Il est contradictoire de prescrire des tâches légales aux associations professionnelles et de grever en même temps le financement de ces tâches (assuré par les cotisations de membre) en l'assujettissant à la TVA.

- Les cabinets médicaux de groupes seraient eux aussi désavantagés par la révision prévue. A titre d'exemple, il est prévu d'abroger la dérogation selon laquelle la redistribution interne de médicaments achetés en commun est exemptée de la TVA.

Tous ces arguments l'emportent sur les avantages de la simplification visée. C'est sans aucun doute une des raisons pour lesquelles l'opposition à la suppression de $l^{\prime}$ «exception Santé» est aussi large. Tant les directeurs cantonaux de la santé que l'organisation faîtière des hôpitaux $\mathrm{H}+$ ou celle des assureurs santésuisse, entre autres, se sont clairement opposés à ce projet. Tous mettent en garde notamment contre l'augmentation massive des coûts de la santé et la hausse inévitable des primes d'assurance qu'elle entraînerait. Le dossier se trouve actuellement dans les mains de la Commission de l'économie et des redevances du Conseil national. Après l'audition lors de laquelle la FMH a encore une fois clairement énoncé sa position, cette commission se penchera sur le contenu du projet lors de ses prochaines séances. Nous suivrons attentivement l'évolution du débat et continuerons à nous engager avec fermeté pour éviter que le domaine de la santé ne subisse un renchérissement dû à la TVA.

Dr Jacques de Haller, président de la FMH 